\title{
Heisenberg Spin Bus as a Robust Transmission Line for Perfect State Transfer
}

\author{
Sangchul Oh, ${ }^{1}$ Lian-Ao Wu, ${ }^{2,3}$ Yun-Pil Shim, ${ }^{4}$ Mark Friesen, ${ }^{4}$ and Xuedong $\mathrm{Hu}^{1}$ \\ ${ }^{1}$ Department of Physics, University at Buffalo, State University of New York, Buffalo, New York 14260-1500, USA \\ ${ }^{2}$ Department of Theoretical Physics and History of Science, \\ The Basque Country University (EHU/UPV), PO Box 644, 48080 Bilbao, Spain \\ ${ }^{3}$ IKERBASQUE, Basque Foundation for Science, 48011 Bilbao, Spain \\ ${ }^{4}$ Department of Physics, University of Wisconsin-Madison, Madison, Wisconsin 53706, USA
}

(Dated: October 5, 2018)

\begin{abstract}
We study the protocol known as quantum state transfer for a strongly coupled antiferromagnetic spin chain or ring (acting as a spin bus), with weakly coupled external qubits. By treating the weak coupling as a perturbation, we find that perfect state transfer (PST) is possible when second order terms are included in the expansion. We also show that PST is robust against variations in the couplings along the spin bus and between the bus and the qubits. As evidence of the quantum interference which mediates PST, we show that the optimal time for PST can be smaller with larger qubit separations, for an even-size chain or ring.

PACS numbers: 03.67.Hk, 03.67.-a., 75.10.Pq
\end{abstract}

Transferring quantum states between qubits is one of the most fundamental tasks in quantum information processing. State transfer can be realized through various approaches. For example, if two qubits have a controllable Heisenberg coupling, a swap gate can be used to exchange their quantum states [1]. For longer range communications, a quantum data bus connecting remote qubits can be employed: examples include phonon modes for trapped ions 2], cavity photon modes for superconducting qubits [3], and spin chains for spin qubits [4, [5]

The feasibility of quantum state transfer through spin chains or lattices depends on the available types of coupling and the degree of controllability. One of the most widely studied schemes is known as perfect state transfer (PST). In this protocol, the bus and qubits are prepared in an initial state; the system is then allowed to evolve freely, with all the spin interactions held constant. State transfer cannot be achieved with perfect fidelity in a uniform spin chain with Heisenberg or XY spin couplings [4], except for individual couplings that are specially engineered and non-uniform [6-10]. The quantum information ordinarily propagates dispersively, with excited states of the system playing a critical role in the evolution. This is in contrast with the adiabatic operating modes of the spin bus, particularly the odd-size bus [5], and it leads to fundamental questions about how the excited states of a spin lattice carry quantum information: whether the propagation of quantum information is limited by the speed of the spin wave [11], and whether the quantum state transfer time is simply proportional to the distance between sender and receiver, in analogy with signal transmission through wires or optical fibers.

In this paper, we return to the question of whether it is possible to implement PST between remote qubits through spin- $1 / 2$ Heisenberg chains or rings with uniform couplings. In this geometry, we show that if the qubit- chain coupling is smaller than the intra-chain coupling (i.e. a spin bus architecture [5]), PST can be achieved with arbitrary accuracy. Further, we show that, despite significant differences in the ground state properties of odd and even-size chains or rings, both systems admit similar types of PST, with fidelities that are insensitive to variations in the individual exchange couplings in the system. These results suggest that we can view a uniformly coupled Heisenberg chain as a robust quantum coherent plug-in device between two spin qubits, like a transmission line between two circuit elements, so that arbitrary quantum states may be swapped after a fixed time determined by the coupling parameters. In addition, we show that quantum interference effects are inherent in the state transfer protocol by demonstrating that the transfer time is not simply proportional to the geometric distance between the source and target qubits. This counterintuitive observation indicates that quantum information transfer through a quantum spin transmission line cannot be fully understood in terms of a classical analog, such as a signal propagating along a metal wire. (a)

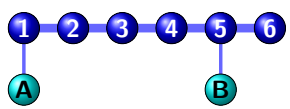

(b)

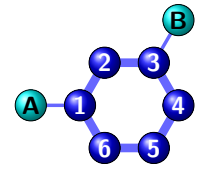

Figure 1: (Color online). PST in the spin bus geometry, where two qubits are coupled to (a) an even or odd spin-chain, or (b) an even size ring. The qubit-bus couplings are taken to be weak compared to intra-bus couplings.

The system we consider consists of two qubits $A$ and $B$ weakly attached to a strongly coupled Heisenberg chain or ring, as shown in Fig. 11 The total Hamiltonian is

$$
H=H_{C}+H_{Q C}
$$


where the Heisenberg Hamiltonian of the chain or ring is

$$
H_{C}=\sum_{i=1}^{N} J_{i} \mathbf{s}_{i} \cdot \mathbf{s}_{i+1} .
$$

We first assume that the chain or ring has uniform antiferromagnetic couplings, with $J_{i}=J_{0}>0$ for $i=$ $1, \cdots, N-1$. The boundary conditions are $J_{N}=0$ for an open chain, or $J_{N}=J_{0}$ and $\mathbf{s}_{N+1} \equiv \mathbf{s}_{1}$ for a ring. The couplings between qubits $A$ and $B$ and the $i$-th and $j$-th spins of the chain are given by

$$
H_{Q C}=J_{A, i} \mathbf{S}_{A} \cdot \mathbf{s}_{i}+J_{B, j} \mathbf{S}_{B} \cdot \mathbf{s}_{j} .
$$

The qubit-chain coupling is assumed to be weak, with $0<\lambda_{\alpha} \equiv J_{\alpha, i} / J_{0} \ll 1$. Here, $\alpha=A, B$, and $\lambda_{\alpha}$ will be used as a perturbation parameter. From now on, we set $J_{0}=\hbar=1$. Note that we have previously studied the low-energy effective Hamiltonian arising from Eq. (11) [12]. Here, we focus on PST between qubits $A$ and $B$, by numerically solving its time evolution.

Let us first define the state transfer protocol for the spin bus geometry. The goal is to transfer an unknown quantum state from qubit $A$ to qubit $B$ through chain $C$ in a fixed time period, during which the spin couplings are held constant. At $t=0$, an initial state of the total system is prepared as $|\Psi(0)\rangle=\left(a\left|0_{A}\right\rangle+b\left|1_{A}\right\rangle\right) \otimes\left|0_{C}\right\rangle \otimes\left|0_{B}\right\rangle$, where qubit $A$ is in a superposed pure state represented by a point on a Bloch sphere with $a=\cos \frac{\theta}{2}$ and $b=\sin \frac{\theta}{2} e^{i \varphi}$, and chain $C$ and qubit $B$ are in their ground states. Then we allow the system to evolve freely, so that $|\Psi(t)\rangle=e^{-i H t / \hbar}|\Psi(0)\rangle$ at time $t$. The reliability of state transfer can be expressed in terms of the fidelity

$$
F_{B}(t)=\left\langle\phi_{T}\left|\rho_{B}(t)\right| \phi_{T}\right\rangle,
$$

where $\rho_{B}(t)=\operatorname{Tr}_{A, C}|\Psi(t)\rangle\langle\Psi(t)|$ is the reduced density matrix of qubit $B$ obtained by tracing out the degrees of freedom of $A$ and $C$ from $|\Psi(t)\rangle$, and $\left|\phi_{T}\right\rangle=a|0\rangle_{B}+$ $b|1\rangle_{B}$ is the target state. We define "state transfer" to occur at time $t_{0}$ when $F_{B}(t)$ attains its first maximum. PST corresponds to the case $F_{B}=1$, where $t_{0}$ must be independent of the initial state. For a uniformly coupled spin chain $\left(\lambda_{\alpha}=1\right), t_{0}$ and the fidelity are both found to depend on the initial state. However, we will show that PST can be attained with arbitrary accuracy in a spin bus geometry with $\lambda_{\alpha}<1$, at a time scale $\sim 1 / \lambda_{\alpha}^{2}$.

Quantum state transfer through odd-size chains.An odd-size Heisenberg open chain has two-fold degenerate ground states, and behaves like a single spin- $1 / 2$ object at low energies [5, 12]. When qubits $A$ and $B$ are weakly coupled to the $i$-th and $j$-th spins of chain $C$, the chain acts as a "central spin." The effective Hamiltonian [12, 13] can be computed to first order in the perturbation $\lambda_{\alpha}$ using the Schrieffer-Wolff transformation [14]:

$$
H_{\mathrm{eff}}=J_{A, i}^{*} \mathbf{S}_{A} \cdot \mathbf{S}_{C}+J_{B, j}^{*} \mathbf{S}_{B} \cdot \mathbf{S}_{C},
$$

where $\mathbf{S}_{C}$ is the central spin operator acting on the twofold degenerate ground states of the chain, $\left\{\left|0_{C}\right\rangle,\left|1_{C}\right\rangle\right\}$. The effective coupling to qubit $A$ is given by $J_{A, i}^{*}=$ $J_{A, i}\left\langle 0_{C}\left|\sigma_{i z}\right| 0_{C}\right\rangle \propto \lambda_{A}$, where $\left\langle 0_{C}\left|\sigma_{i z}\right| 0_{C}\right\rangle$ represents the local magnetic moment at the $i$-th site of the chain. Due to the antiferromagnetic nature of the bare couplings, the effective couplings $J_{\alpha, i}^{*}$ alternate in sign as a function of the site position, $i$. From now on, we assume qubits $A$ and $B$ are either both attached to even nodes or both attached to odd nodes of the chain, so that their effective couplings are both ferromagnetic or both antiferromagnetic. If the qubits are attached to mixed nodes on the chain, we find that PST is not possible. For simplicity here, we take $J_{A, i}=J_{B, j}$, so that $\lambda_{\alpha}=\lambda$.

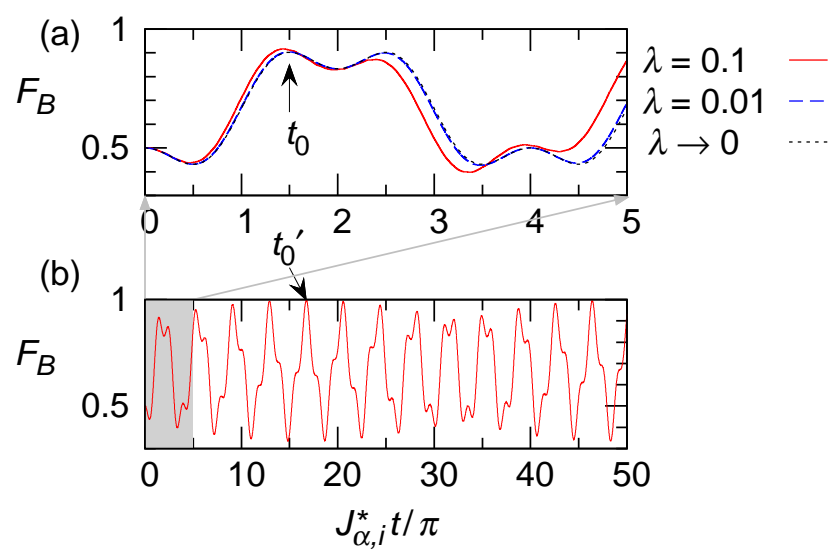

Figure 2: (Color online). PST fidelity $F_{B}(t)$ vs. time $t$ with an odd-size spin bus, for short (a) and long (b) time evolutions. The chain size here is $N=5$, and the initial state of qubit $A$ is $\left|\psi_{A}\right\rangle=\frac{1}{\sqrt{2}}\left(\left|0_{A}\right\rangle+\left|1_{A}\right\rangle\right)$. In panel (b), $\lambda=0.1$.

If switching of the bare couplings $J_{\alpha, i}$ is allowed during the evolution period, quantum state transfer from $A$ to $B$ can be achieved simply, through sequential swap operations involving the bus [5]. In the conventional PST protocol, however, the couplings must remain fixed. Under this condition, it is impossible to transfer a quantum state from qubit $A$ to qubit $B$ perfectly, based on the evolution of the effective Hamiltonian (5). In this case, the fidelity can be computed exactly:

$$
F_{B}(t)=\frac{1+\cos \theta}{2}+\frac{\sin ^{2} \theta}{4} f(t)+\frac{(1-\cos \theta)^{2}}{4} g(t),
$$

where $f(t)=(5+4 \cos 6 \tau+3 \cos 4 \tau-12 \cos 2 \tau) / 18$ and $g(t)=(7+2 \cos 6 \tau-3 \cos 4 \tau-6 \cos 2 \tau) / 18$ with $\tau \equiv J_{\alpha, i}^{*} t / 4 . \quad F_{B}(t)$ attains its initial maximum at $t_{0} \sim 1 / J_{\alpha, i}^{*} \sim 1 / \lambda_{\alpha}$, as shown in Fig. 2 (a). However, the maximum fidelity and the optimal time both depend on the state to be transferred, as shown in Fig. 3 .

While PST is not attainable using the effective Hamiltonian (5), this represents only the lowest order approximation to the full Hamiltonian (1) in the Schrieffer-Wolff transformation. When we compute the time evolution of 

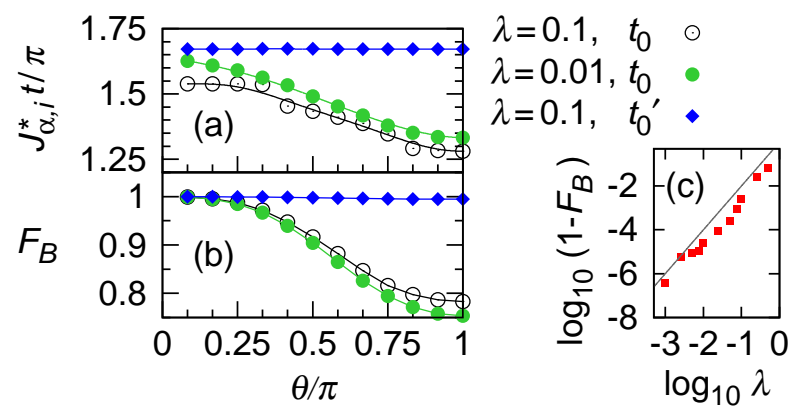

Figure 3: (Color online). (a) Optimal times for state transfer, $t_{0}$ and $t_{0}^{\prime}$ (scaled by $J_{\alpha, i}^{*} / \pi \sim \lambda$ ), as a function of the polar angle $\theta$ of the initial state, with an odd-size bus. (b) Maximum fidelity $F_{B}$ as a function of $\theta$. (c) Log-log plot of infidelity $\left[1-F_{B}\left(t_{0}^{\prime}\right)\right]$ vs. $\lambda$. The line represents $\lambda^{2}$.

the full Hamiltonian, we find that PST becomes possible. Figure 2 shows the fidelity for short and long time periods for various $\lambda$. In the limit $\lambda \rightarrow 0$, Eq. (5) becomes an excellent approximation and the fidelity follows Eq. (6). On the other hand, as shown in Fig. 2 (b), for $\lambda=0.1$, the higher order terms have a non-trivial effect on the time evolution, leading to a fidelity that approaches 1 at a new optimal time $t_{0}^{\prime} \sim 1 / \lambda^{2}$, which is about a factor $1 / \lambda$ longer than $t_{0}$. More importantly, both the maximum fidelity and the optimal time $t_{0}^{\prime}$ are found to be independent of the initial state, as shown in Figs. 3(a) and (b), so that an unknown quantum state can be transferred perfectly. Figure 3(c) shows a log-log plot of $\left[1-F_{B}\left(t_{0}^{\prime}\right)\right]$ vs. $\lambda$. The infidelity has an approximate $\lambda^{2}$ dependence, suggesting that the second order terms play a key role in PST. In short, for an odd-size spin bus geometry, $\left[1-F_{B}\left(t_{0}^{\prime}\right)\right] \sim \lambda^{2}$ and $t_{0}^{\prime} \sim 1 / \lambda^{2}$, so that PST can be achieved with arbitrarily high accuracy for smaller $\lambda$, albeit at the cost of a longer $t_{0}^{\prime}$.

Quantum state transfer through even-size chains or rings. - Even numbers of spins with antiferromagnetic couplings are compensated, so the ground state of an even-size spin bus (chain or ring) is nondegenerate, and has no net magnetization. When qubits are weakly attached, an even-size chain or ring mediates an indirect exchange coupling between them. The effective Hamiltonian up to second order in the bare coupling strength is 12 ]

$$
H_{\mathrm{eff}}=e_{0}\left|0_{C}\right\rangle\left\langle 0_{C}\right|+J_{i, j}^{*} \mathbf{S}_{A} \cdot \mathbf{S}_{B},
$$

where the effective coupling is given by

$$
J_{i, j}^{*}=\frac{J_{A, i} J_{B, j}}{2} \sum_{n \neq 0} \frac{\left\langle 0_{C}\left|\sigma_{i \mu}\right| n_{C}\right\rangle\left\langle n_{C}\left|\sigma_{j \mu}\right| 0_{C}\right\rangle}{e_{0}-e_{n}} .
$$

Here $e_{n}$ and $\left|n_{C}\right\rangle$ are the eigenvalues and eigenstates of an isolated $(\lambda=0)$ even-size spin bus, which we obtained numerically by solving the full spectrum of Hamiltonian (22). Again we take $J_{A, i}=J_{B, j}$, so that $J_{i, j}^{*} \sim \lambda^{2}$. The time evolution of Hamiltonian (7) leads to a direct swap operation between qubits $A$ and $B$, and to PST. In this case, the antiferromagnetic and ferromagnetic effective couplings are equally effectual for PST (in contrast with their ability to mediate ground state entanglement [12]). The absolute value of $J_{i, j}^{*}$ determines the speed of the swap operation. The speed and fidelity of such quantum state transfer has previously been studied in a related but distinct geometry [15].

In the limit of $\lambda \ll 1$, Eq. (7) forms an excellent approximation to the full Hamiltonian. The fidelity of qubit $B$ is then given by

$$
F_{B}(t)=1-\frac{1}{2} \sin ^{2} \theta \cos ^{2}\left(\frac{J_{i, j}^{*} t}{2}\right) .
$$

In contrast with the case of an odd-size chain, PST now occurs at the first fidelity maximum, given by $J_{i, j}^{*} t_{0}=\pi$, and it is independent of the initial state. However, in this case, $t_{0} \sim 1 / \lambda^{2}$, similar to $t_{0}^{\prime}$ for an odd-size chain.
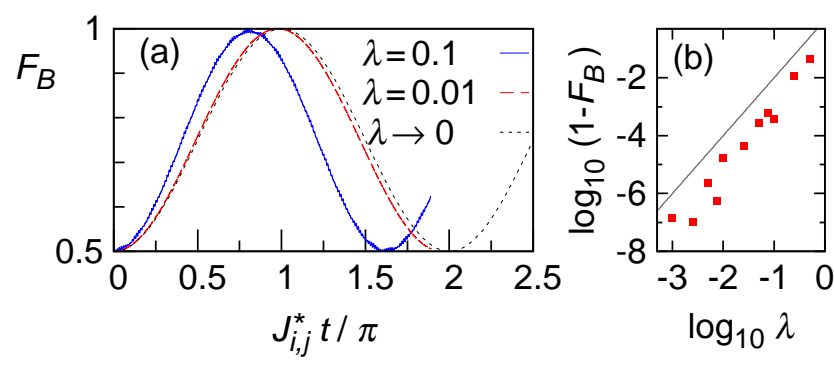

Figure 4: (Color online). (a) Fidelity $F_{B}(t)$ as a function of time $t$ when qubits $A$ and $B$ are attached to the opposite ends of an even bus of size $N=4$. The initial state of qubit $A$ is $\left|\psi_{A}\right\rangle=\frac{1}{\sqrt{2}}(|0\rangle+|1\rangle)$. (b) Infidelity $\left[1-F_{B}\left(t_{0}\right)\right]$ vs. $\lambda$. The line represents $\lambda^{2}$.

Going beyond the lowest order effective Hamiltonian (7), we can obtain exact numerical solutions using the full Hamiltonian, with the results shown in Fig. 4. While panel (a) shows that $t_{0} \sim 1 / \lambda^{2}$, panel (b) shows that PST occurs in the limit $\lambda \rightarrow 0$, with $\left[1-F_{B}\left(t_{0}\right)\right] \sim \lambda^{2}$. Both results have a direct correspondence with the oddsize bus geometry. We can therefore draw several conclusions. First, despite having very distinct ground states, even and odd-size buses both allow PST, over time scales of order $1 / \lambda^{2}$. The similar behaviors seem to derive from the second order terms in the Schrieffer-Wolff transformation. Second, for larger $\lambda$, when higher order terms become important, PST is increasingly degraded. In this sense, it appears that the second order terms are responsible for PST.

The time evolution of the even-size bus takes a simpler form than the odd-size bus, and the quantum interference is less pronounced. However, we now probe aspects of the evolution that are distinctly quantum in origin. We study the dependence of PST on the separation distance between the attached qubits, for the case of an even-size 

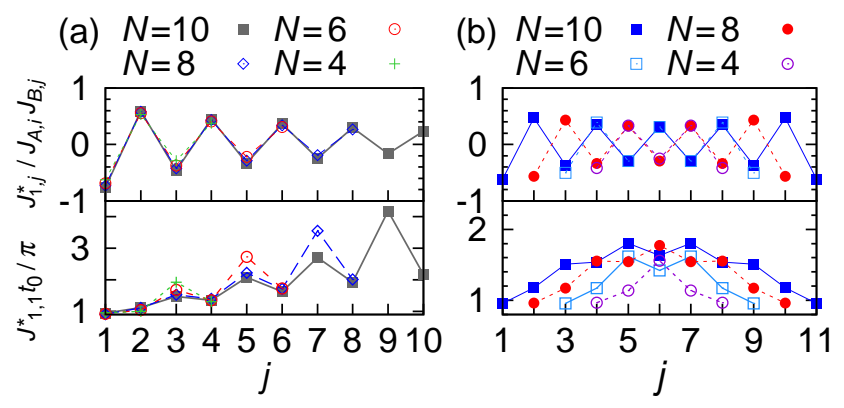

Figure 5: (Color online). Effective couplings $J_{1, j}^{*}$, scaled by $J_{A, 1} J_{B, j}$, and the corresponding optimal times $t_{0}$, scaled by $\pi / J_{1,1}^{*}$, as a function of position $j$ for even-size (a) chains and (b) rings of size $N$. Here, $J_{A, 1}=J_{B, j}=0.1$. In (b), plots with $N=4,6,8$ are shifted to the center, for comparison.

bus. Here qubit $A$ is attached to the first node of the chain or ring, $i=1$, while qubit $B$ is attached to node $j=1,2, \ldots, N$ (see Fig. 1). For an open chain bus, the optimal time is found to increase as an oscillatory function of $j$, as shown in Fig. [5(a). This leads to the striking observation that it can take less time to transfer a quantum state to a further qubit than a closer qubit.

Similar behavior is observed for an even-size ring bus, where an additional even-odd parity effect emerges, as shown in Fig. 5 (b). This depends on the size of the chain: $N=2 \times 2 n$ or $2 \times(2 n+1)$. To see this, we first note that the ring has rotational symmetry. We might expect the transfer time to be maximized for a pair of antipodes, and this is true when $N=2 \times 2 n$. However, when $N=2 \times(2 n+1)$, the PST time is maximized for the neighbors of the antipode. Note that the effective coupling between antipodes is antiferromagnetic if $N=$ $2 \times(2 n+1)$, and ferromagnetic if $N=2 \times 2 n$. We conclude that quantum state transfer can be faster over longer distances than shorter distances. Such effects arise, in part, from quantum fluctuations of the bus eigenstates, and are distinctly quantum in origin.

So far we have focused on PST through spin buses with uniform Heisenberg exchange couplings. We have also studied how the fidelity of PST is influenced by random variations of the exchange couplings in the bus. Consider the even-chain case as an example. The form of the effective second order exchange interaction, shown in Eqs. (7) and (8), does not change in the presence of small exchange variations in the bus, as long as the ground state of the bus remains nondegenerate. Thus PST should still be feasible, and its fidelity should not change if the PST time $t_{0}$ (or $t_{0}^{\prime}$ ) is calibrated to account for the variations in the effective qubit coupling strength $J_{i, j}^{*}$. Without calibration, PST error should grow quadratically due to the sinusoidal time dependence of the PST fidelity (see Fig. (5). Figure.6 clearly shows that after calibration, the PST fidelities for both even and odd size buses are indeed insensitive to variations in the exchange coupling. With-

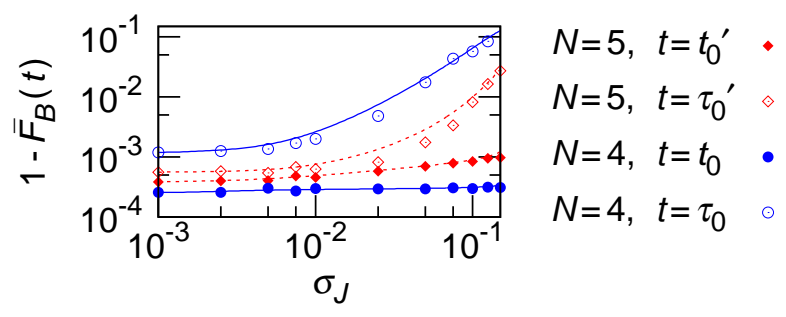

Figure 6: (Color online). Average (over 100 random configurations with variance $\sigma_{J}$ ) PST infidelity $1-\bar{F}_{B}(t)$ with a 4- or 5-node bus as a function of exchange variation, represented by the variance $\sigma_{J}$ of a Gaussian distribution around the target $J_{0}$. Here $\tau_{0}\left(\tau_{0}^{\prime}\right)$ is the PST time for the case of uniform exchange couplings, while $t_{0}\left(t_{0}^{\prime}\right)$ refers to the PST time calibrated for non-uniform coupling strengths.

out calibration, the PST error grows quadratically with timing error as expected. For variations $\delta J / J_{0}<0.01$, fidelity in both cases hardly increases compared to the perfect, uniform coupling result.

In conclusion, we have demonstrated perfect state transfer in a system where it was not expected: a strongly coupled Heisenberg chain or ring with uniform couplings. We have pinpointed the source of the effect in the second order terms in a Hamiltonian expansion, assuming perturbative couplings to the qubits. We have also shown that our PST protocol does not require precise engineering of the intra-bus exchange couplings, and that external qubits do not have to be coupled to the end nodes of the bus - they can be anywhere on an even bus, or on the same type of nodes (both even or both odd) on an odd bus. Moreover, we showed that for even-size chains or rings, the optimal time for PST is not linearly proportional to the geometric distance between sender and receiver, emphasizing the fundamental differences between quantum and classical signal transmission. Overall, our results point to a truly robust coherent plug-in device for perfect quantum state transfer between remote spin qubits. The results presented here may be tested using existing technologies such as spin chains on a metal surface [16], or with quantum dot 17], molecular [18], or NMR qubits [19].

This work was supported by the DARPA QuEST through AFOSR and NSA/LPS through ARO. L.A.W. was supported by a Ikerbasque Foundation Start-up, the Basque Government (grant IT472-10) and the Spanish MEC (Project No. FIS2009-12773-C02-02).

[1] D. Loss and D. DiVincenzo, Phys. Rev. A 57, 120 (1998).

[2] J. I. Cirac and P. Zoller, Phys. Rev. Lett. 74, 4091 (1995).

[3] A. Blais, R.-S. Huang, A. Wallraff, S. M. Girvin, and R. J. Schoelkopf, Phys. Rev. A 69, 062320 (2004).

[4] S. Bose, Phys. Rev. Lett. 91, 207901 (2003); Contem. 
Phys. 48, 13 (2007).

[5] M. Friesen, A. Biswas, X. Hu, and D. Lidar, Phys. Rev. Lett. 98, 230503 (2007).

[6] M. Christandl, N. Datta, A. Ekert, and A. J. Landahl, Phys. Rev. Lett. 92, 187902 (2004).

[7] L.-A. Wu, Y.-X. Liu, and F. Nori, Phys. Rev. A 80, 042315 (2009).

[8] P. J. Pemberton-Ross and A. Kay, Phys. Rev. Lett. 106, 020503 (2010).

[9] N. Y. Yao et al., arXiv:1011.2762,

[10] S. Yang, A. Bayat, and S. Bose, arXiv:1101.3790.

[11] J. Eisert and D. Gross, Phys. Rev. Lett. 102, 240501 (2009).

[12] S. Oh, M. Friesen, and X. Hu, Phys. Rev. B 82,
140403(R) (2010)

[13] Y.-P. Shim, S. Oh, X. Hu, and M. Friesen, arXiv:1012.0565

[14] J. R. Schrieffer and P. A. Wolff, Phys. Rev. 149, 491 (1966).

[15] L. Campos Venuti, C. Degli Esposti Boschi, and M. Roncaglia, Phys. Rev. Lett. 99, 060401 (2007).

[16] C. F. Hirjibehedin, C. P. Lutz, and A. J. Heinrich, Science 312, 1021 (2006).

[17] J. R. Petta et al., Science 309, 2180 (2005).

[18] G. A. Timco et al., Nature Nanotech. 4, 173 (2009).

[19] P. Cappellaro, C. Ramanathan, and D. G. Cory, Phys. Rev. Lett. 99, 250506 (2007). 